\title{
Collaborative Networks - Premises for Exploitation of Inter-Organizational Knowledge Management
}

\author{
Marinela MIRCEA \\ The Bucharest University of Economic Studies, Romania \\ mmircea@ase.ro
}

Inter-organization knowledge management in the context of collaborative networks is a critical activity for business success. During the evolution of collaborative network specific technologies, increasingly performant instruments were created to exploit this knowledge. As a development cycle, inter-organizational knowledge is built on the foundation of information and data owned by the participants in the collaborative networks. One of the most widely used instruments to exploit this data and knowledge, with the purpose of creating new knowledge, is Data Mining. In the context of this paper, data mining is the process of discovering patterns and hidden relations in very large data collections, stored in data banks or data bases. Because only in extremely rare cases reading data tables record by record leads to the discovery of useful patterns, the information must be processed automatically, process known as Knowledge Discovery. Knowledge Discovery is a component that combines the power of computers with a human operator that has the ability to find the visual patterns revealed by the system. Using an automated data mining system, the computer finds the existing informational patterns and the human factor (the analyst) evaluates those patterns and picks the ones that are really relevant for the current analysis. Considering the current technological context, where storage devices are more and more accessible and performant, the storage capacity is no longer a barrier preventing storage of all required data. Exploitation of inter-organizational knowledge in collaborative networks leads the research to the field of business intelligence applied even on social environment. This approach belongs in literature to the general branch of social business intelligence.

Keywords: Collaborative Networks, Information Technology, Inter-Organizational Knowledge, Knowledge Management, Knowledge Discovery, Data Mining

1 Introduction

The importance of knowledge and organizational networks in obtaining a competitive advantage on the market is recognized both by theoreticians and by practitioners as well. The increase of the number of collaborative networks, the accent on knowledge within the society based on knowledge and innovation come to support those who want to adhere to new organizational forms that would lead to obtaining success on the global market.

Knowledge is known as one of the most important assets of management within organizations, as knowledge allows organizations to use and develop resources, to increase the competitive ability and to obtain a substantial competitive advantage [1]. Knowledge also represents an important source that allows nations, organizations, and persons to obtain benefits, such as: learning improvement, innovation, and decision making. Any organization, public or private, needs a knowledge management process in order to obtain the best performances [2].

In global economy, the strong competition, the frequent changes on the market, the higher and higher demands concerning quality, lead to the necessity of new organizational forms. Organizational networks are acknowledged as organizational forms characterized by an increased flexibility and that may lead to obtaining the competitive advantage on the market. Apart from the potential advantages, organizational networks are also confronted with problems and challenges that are particularly connected to the complexity of the collaborative environment. 
Inter-organizational knowledge can be defined as an explicit set of knowledge that is formalized and created by organizations [3]. The interactions within the network allow organizations to develop the collaborative and relational tacit knowledge and to generate inter-organizational tacit knowledge that can be capitalized within the interorganizational memory [4]. The interorganizational knowledge allows organizations to develop distinctive abilities, which may lead to the increase of the innovation ability.

Inter-organizational knowledge management is a complex process due to both the nature of knowledge (intangible, dynamic, intrinsic) and to the collaborative environment as well. One of the major challenges of interorganizational knowledge management is its sharing among the members of the organizational network. The knowledge sharing depends on a series of factors, such as: the security of the communication channel, the organizational culture of the participants and their roles, the nature of knowledge (tacit and explicit; formal and informal), the organizational structure, and the support offered by the information and communications technology (ICT).

Interoperability also represents another challenge within the inter-organizational knowledge management. The existence of certain heterogeneous knowledge management systems within different partner organizations that are not able to communicate and to integrate themselves, leads to the limitation of reusing the interorganizational knowledge (formalized explicit knowledge) [1].

The main purpose of the paper is represented by the analysis of the knowledge management process within organizational networks, with an emphasis on the changes produced by the collaborative environment. Throughout the paper the importance and necessity of an inter-organizational knowledge management is emphasized, a comparative analysis of certain aspects of intra and inter-organizational knowledge is performed and the collaborative characteristics that influence the stages of the knowledge management process are identified.

\section{Inter-Organizational Knowledge in Collaborative Networks}

The analysis of the knowledge management process involves the recognition of the types of knowledge and of the means in which the collaboration influences the stages of their management process. The explicit or coded knowledge is the knowledge that is transmitted through a formal, systemic language and that is sent as data, scientific formulas, specifications or manuals [5]. In such case, both the communications technologies and the knowledge management systems of organizations influence their management as well. Insuring the knowledge management systems' interoperability is also a critical fact for the success at the level of network.

Tacit knowledge is personal and difficult to formalize. Tacit knowledge is deeply rooted in action, procedures, routine, commitments, ideals, values and emotions [5]. Their transfer is much more difficult to accomplish between organizations. The interactions within the networks, the organizational culture, and the communications abilities are only a few of the factors that influence the management of these types of knowledge. Moreover, according to [6], the lack of certain common goals, the significant differences in the corporate culture, the competition between partners, the lack of certain cooperation rules, the inadequate coordination and the opportunistic behavior lead to difficulties and restrictions within the inter-organizational use of knowledge.

In organizational networks trust represents a catalyst that enables strategic business interactions and knowledge sharing between organizations [7], [8]. Trust symbolizes the positive psychological state in which you believe in the goodwill and integrity of the correspondent regarding the support of the promise to obtain common results. Trust is the fundamental condition necessary for eliminating incertitude within the interaction processes [9]. 
Inter-organizational knowledge presents some differences compared to intraorganizational knowledge, due to the complexity of the collaborative environment in particular. Table 1 displays some aspects regarding the intra and inter-organizational knowledge. The differences are not only limited to the aspects in the table. Moreover, the structure of the network, the form of organization (partnerships on long or short term), the size of the network, as well as other aspects, influence the interorganizational knowledge, leading to certain differences within the stages of the management process.

Table 1. Intra and inter-organizational knowledge aspects

\begin{tabular}{|c|c|c|}
\hline Aspects & Intra-organizational & Inter-organizational \\
\hline $\begin{array}{l}\text { Geographical } \\
\text { area }\end{array}$ & $\begin{array}{lr}\text { Teams, } & \text { national } \\
\text { organization, } & \text { multinational } \\
\text { organization. } & \end{array}$ & Organizational network \\
\hline Barriers & Formal, hierarchic. & Structural, communication, cultural. \\
\hline Creation & $\begin{array}{l}\text { The knowledge creation is } \\
\text { performed within } \\
\text { organization. Many a time, } \\
\text { the knowledge is created in } \\
\text { specialized departments } \\
\text { (departments of research } \\
\text { and development). }\end{array}$ & $\begin{array}{l}\text { The knowledge creation takes place in an } \\
\text { unsecure environment. There is a possibility } \\
\text { that within the process, the partner's goals or } \\
\text { behavior might change and, furthermore, } \\
\text { their collaboration relationships. Through } \\
\text { interactions and the reuse of available } \\
\text { knowledge at the level of network, the } \\
\text { possibility of creation of new knowledge is } \\
\text { higher. }\end{array}$ \\
\hline Transfer & $\begin{array}{l}\text { It is accomplished through } \\
\text { the compliance with the } \\
\text { intellectual property rights. } \\
\text { It overlaps with the } \\
\text { knowledge change within } \\
\text { organization. }\end{array}$ & $\begin{array}{l}\text { It is influenced by the characteristics of the } \\
\text { network, such as: culture, type of alliance, } \\
\text { available ICT. It shows common } \\
\text { characteristics with many of the knowledge } \\
\text { acquisition processes [10]. }\end{array}$ \\
\hline Share & $\begin{array}{l}\text { Takes place in keeping with } \\
\text { the roles, security and } \\
\text { integrity of the knowledge } \\
\text { management system within } \\
\text { organization. }\end{array}$ & $\begin{array}{l}\text { Takes place when the partners manage to } \\
\text { cultivate trust and to build long term } \\
\text { partnerships. }\end{array}$ \\
\hline Storage & $\begin{array}{l}\text { It is carried out in } \\
\text { databases/knowledge bases, } \\
\text { according to the } \\
\text { organization's strategy. }\end{array}$ & $\begin{array}{l}\text { It is carried out according to the agreements } \\
\text { within the network and to the available } \\
\text { technology. Furthermore, the existence of } \\
\text { interoperability at the level of knowledge } \\
\text { management systems is necessary. }\end{array}$ \\
\hline $\begin{array}{l}\text { Use/ } \\
\text { Reuse }\end{array}$ & $\begin{array}{l}\text { Leads to the development of } \\
\text { new } \\
\text { products/services/added } \\
\text { value. }\end{array}$ & $\begin{array}{l}\text { Leads to the development of more new } \\
\text { knowledge, through the interaction of the } \\
\text { involved partners, trust and common goals. }\end{array}$ \\
\hline
\end{tabular}

In long-term organizational networks, knowledge represents the decisive base for the intelligent and competent performance of partners, and inter-organizational knowledge management becomes a new paradigm of strategic management [6]. 
3 Inter-Organizational Knowledge Management

Inter-organizational knowledge management requires an adequate technology (platforms, applications, and instruments), support infrastructure (processes, network roles) and human capital (digital abilities, collaboration competences and reflexive practical abilities). The adequate technology depends on the purpose of the organizational network, on the digital abilities of its members, as well as on the institutionalization degree of their processes [11]. Starting from the stages of the knowledge management process identified by Schwartz [12] and from the support ICT is able to offer in every stage of the management process, figure 1 presents the knowledge management process in the context of organizational networks.

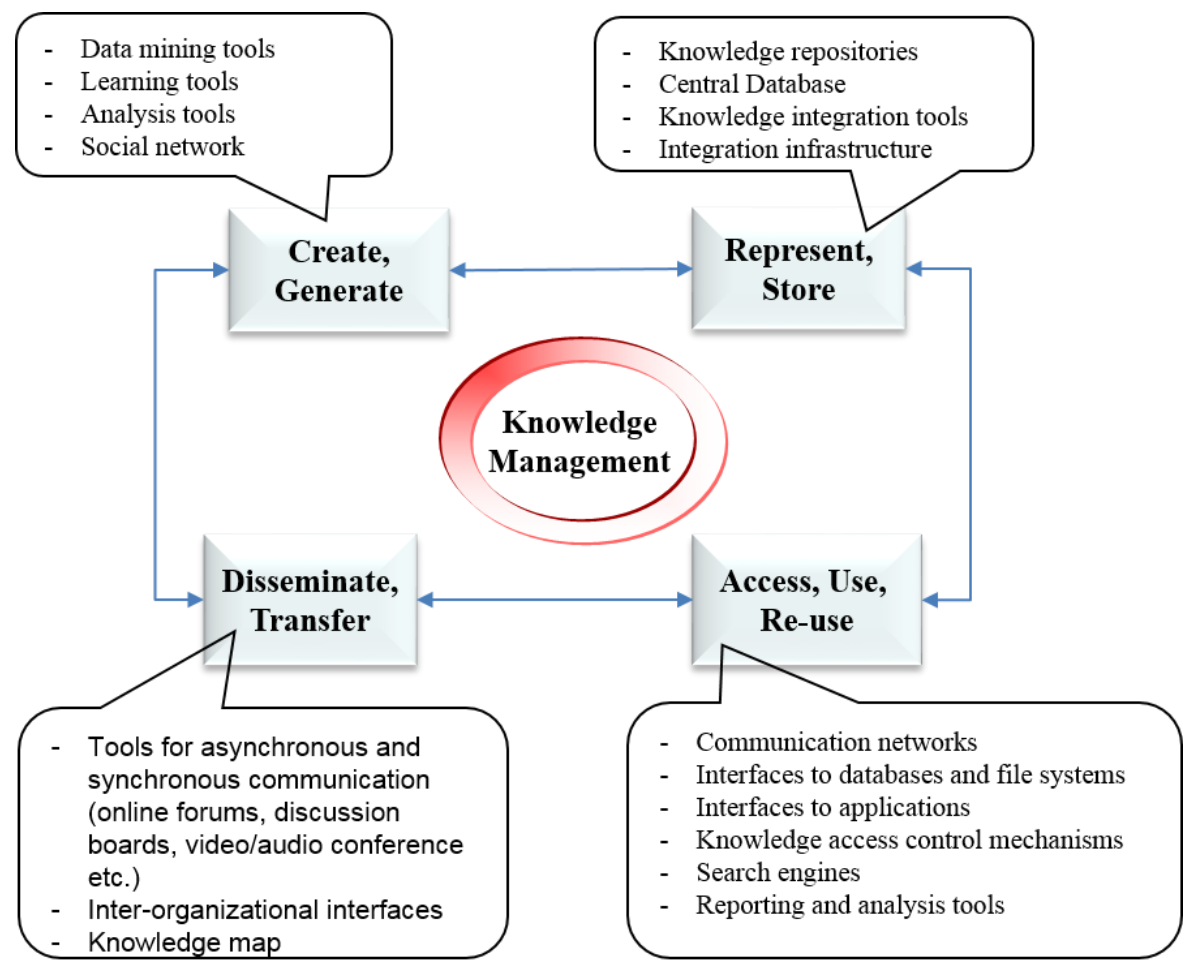

Fig. 1. Knowledge management in collaborative networks

\subsection{Knowledge Creation/Generation}

The knowledge generation process can be accomplished through knowledge acquirement or through knowledge creation [10]. In order to effectively produce a creation process of inter-organizational knowledge, the partners involved in the network must line up their knowledge bases during the knowledge sharing process [13]. Irrespective of the knowledge nature (tacit, explicit), knowledge creation involves the necessity of a common goal. The common goal and the connections are elementary for allowing cooperation within the organizational network. The interactions that take place within the network are essentially connection elements within the network [14]. Once the partnership is established, organizations must take into account the knowledge assignation for the creation and sharing activities, as well as the behavioral aspects regarding the partners' orientation concerning the future value of the results obtained together [13].

\subsection{Knowledge Representation/Store}

Knowledge representation is the process of representation in a graphical form and that can use different pieces of information and communication codes (natural language, figures, drawings, photographs, flux diagrams). The explicit knowledge, until the 
moment of representation, is informal knowledge. The only stored information is the one necessary for identifying the persons and places where knowledge is located [10]. Knowledge storage depends on the technology available within the network. Knowledge can be stored in databases or knowledge bases. Within the organizational network there can exist different knowledge representation languages, different management systems, questioning the interoperability at the level of network.

\subsection{Knowledge Access/Use/Reuse}

Knowledge access is performed according to the agreements within the organizational network and to the available systems. Organizations allow the taking over of interorganizational knowledge and of knowledge that can be reused in order to support the five stages of the knowledge management process. Thus, the reusable interorganizational knowledge network is developed in order to allow member organizations to reuse inter-organizational knowledge that is stored in the knowledge reservoirs of organizations [3].

\subsection{Knowledge Dissemination/Transfer}

Knowledge transfer is an interactive process through which organizations accumulate and develop new knowledge; it allows business partners to detect and understand business problems and to develop viable solutions [9]. The knowledge transfer can be accomplished through different mechanisms: formal or informal [15]; coded or personalized; individualized or institutionalized [16]. Wagner claims that sharing knowledge within an inter-organizational network allows the creation of more diverse knowledge than sharing within an organization [17]. The transfer of knowledge shall be accomplished through a user interface, according to the used mechanism and the agreements established at the level of network.

In case the knowledge is tacit, the communication is best carried out through socialization or through the facilities offered by the multimedia communication technology. The effectiveness and efficiency of the knowledge transfer processes is affected by the assimilation capacity of the receiver [10]. In order to carry out a better knowledge transfer, both the receiver and the sender must have a common fund of knowledge (the existence of a redundancy). If there is no common fund of knowledge, the receiver will not have the possibility to understand the transferred content, and the process of information is useless. At the opposite side, if the receiver already contains the transferred information, then it already represents knowledge for the receiver.

\section{Collaborative Networks and Social Business Intelligence}

Inter-organization knowledge management in the context of collaborative networks involves the existence of specific patterns, built on the foundation of performance key indicators (PKI - Performance Key Indicators) of the business. A management model that brings collaborative knowledge into the social business intelligence may be based on six independent operations (figure 2) [18].

- Strategic transformation of performance key indicators

Each organization (part of the collaborative network or not) computes PKI for its field of activity. They are measured for later use, being a starting point for social business knowledge management. Some of the indicators are not fitted for measuring by social media, because there is no relation between them and social media. Another part of the key indicators are measured through internal systems and a third part is measured by social media. PKI measured by the media establishes the interest categories to be studied from the perspective of interorganizational knowledge management. Also, in collaborative context, collected information indicates if it is possible or not to measure the indicators using the available data from the media environment. 


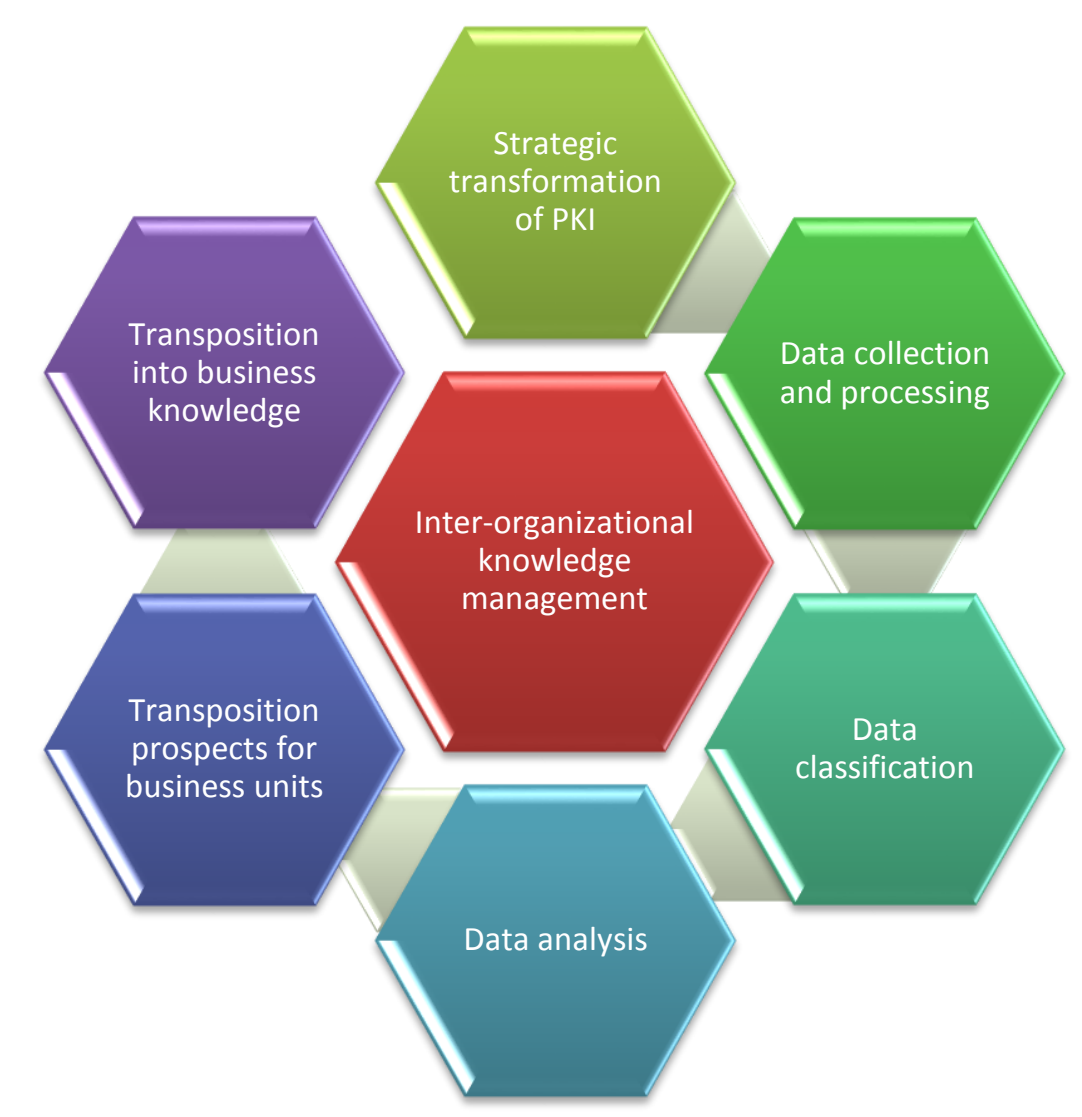

Fig. 2. Model in six steps for inter-organizational knowledge management

- Data collecting and pre-processing

Collection of data is carried out using key words in social media where the organization is mentioned. This involves not only mentions of the organization name, but also products or services provided. Queries to find key words related to brand, products and services will return unstructured data from multiple social media platforms. This data will be pre-processed in the next stage of business intelligence. There is a difference from data provided by a usual business intelligence system, where there are not so many unstructured data.

Collected data consists of media messages, in various formats (CSV, JSON, XML etc.) depending on the media network where they were extracted from. Each source may use its own type of messages and often the number of data attributes varies. Each message must be parsed and structured in the same format. The last step in the data pre-processing is elimination of spam. After pre-processing is over, data will be structured and ready to be classified.

\section{- Data classification}

The third step in the proposed model classifies the messages extracted from social media. The purpose is to divide the messages in cluster categories considering the interests of the organization. The analysis of the categories may provide a starting point for choosing the company strategy for the future.

\section{- Data analysis}

After data from media environments were collected, cleaned and structured, the fourth stage may start. In this stage data from collected messages is transformed into information. Depending on interest domains of the organization, a lot of relations may be analyzed; also, client opinion is point of interest. Starting from the categories established in the previous step, sentiment analysis applied on the messages may discover the public opinion regarding the organization products. This will lead to a better perception of improvements for products and services. The most important information comes from combining media measurements - number of mentions, 
sentiments, messages originating from certain regions - with company PKI - sales volume, market share, client satisfaction and number of clients.

\section{- Applying perspectives to business} units

The previous analysis has revealed some PKI with low performance. Since each indicator belongs to a certain department, its manager may decide on how to improve the situation and adopt strategies to improve current position. Information gained from analysis is sent to the departments with low performance. The perspectives regarding products and services belonging to low performance departments are sent to the development department. Departments with knowledge and experience in finding out why some PKI have low performance are the ones that develop actions to improve the situation.

\section{- Apply knowledge into business}

The last step of the social business intelligence procedure involves execution $\mathrm{pf}$ plans aiming to improve low performance PKI, as revealed by media domains. An organization (whether in collaborative context or not) may decide to review its products based on client feedback and suggestions provided by social business intelligence procedure. Also, it may intervene in the media environment where the results were unsatisfactory. Additionally, the organization may promote some product section where data was missing or reactions were negative. All these intervention policies from the organization are translated into applying the results of knowledge model into business.

Although it is not a part of the six stage model for inter-organizational knowledge management, verification of the results is an important step. The requirements of the social business intelligence procedure may be verified considering: access to social networks, identification of social networks that mention the organization, identification of business related data volume, classification of messages on social networks in categories, clustering the messages on subjects related to PKI, evaluation of organization reputation in social environment, evaluation of relations between social media organization PKI etc.

\section{Conclusions}

The society based on knowledge, the new organizational forms, the global market, the development of the information and communications technology, are the pillars of the continuous development and innovation. The knowledge associated with new organizational forms and with appropriate means of management may lead to agility and to gaining the competitive advantage on the market. However, their embrace does not guarantee organizational success without an adequate analysis of the ICT support. The present paper points out certain aspects regarding inter-organizational knowledge within organizational networks. The paper represents a starting point towards a more detailed analysis of interorganizational knowledge management and of the appropriate ICT support.

\section{Acknowledgment}

This paper was co-financed from the European Social Fund, through the Sectoral Operational Programme Human Resources Development 2007-2013, project number POSDRU/ 159/1.5/S/138907 "Excellence in scientific interdisciplinary research, doctoral and postdoctoral, in the economic, social and medical fields - EXCELIS", coordinator The Bucharest University of Economic Studies.

A shorter version of this paper was presented at the 14th International Conference on Informatics in Economy (IE 2015), May 1-3, 2015.

\section{References}

[1] N. Leung, S.K. Lau and J. Fan, "Enhancing the Reusability of InterOrganizational Knowledge: an Ontology-Based Collaborative Knowledge Management Network," Electronic Journal of Knowledge 
Management, vol. 7, no. 2, pp 233 - 244, 2009.

[2] K.A. Al-Busaidi, "A Framework of Critical Factors to Knowledge Workers' Adoption of Inter-organizational Knowledge Sharing Systems," Journal of Organizational Knowledge Management, vol. 2013, pp. 1-11, 2013.

[3] N.K.Y. Leung, S.K. Lau and J. Fan, "An Ontology-Based Knowledge Network to Reuse Inter-Organizational Knowledge," in 18th Australasian Conference on Information Systems, Toowoomba, 2007, pp. 896-906.

[4] I. Zouaghi, "Tacit Knowledge Generation and Inter-Organizational Memory Development in a Supply Chain Context," Systemics, Cybernetics and Informatics, vol. 9, no. 5, pp. 77-85, 2011.

[5] E. Shijaku. Knowledge creation and sharing in an organization: An empirical analysis of the New Product Development process. Internet: https://www.theseus.fi/bitstream/handle/ 10024/21190/Shijaku_Elio.pdf?sequence $=1,2010$ [February 20, 2015]

[6] T. Blecker and R. Neumann, "Interorganizational Knowledge Management: Some Perspectives for Knowledge Oriented Strategic Managment in Virtual Organizations," Interorganizational Knowledge Management, pp. 63-83, 2000.

[7] J.H. Cheng, "Inter-organizational relationships and information sharing in supply chains," International Journal of Information Management, vol. 31, no. 4, pp. 374-384, 2011.

[8] S. Hoejmose, S. Brammer and A. Millington, "Green supply chain management: the role of trust and top management in B2B and B2C markets," Industrial Marketing Management, vol. 41, no. 4, pp. 609-620, 2012.

[9] Y.H. Chen, T.P. Lin and D. Yen, "How to facilitate inter-organizational knowledge sharing: The impact of trust," Information \& Management, vol. 51, pp. 568-578, 2014.
[10] Y. Jarrar, M. Zairi and G. Schiuma. Defining Organisational Knowledge: A Best Practice Perspective. Internet: http://ecbpm.com/files/Knowledge\%20 Management/Defining\%20Organisationa 1\%20Knowledge.pdf, 2010 [February 20, 2015]

[11] M. Velasco. Knowledge transfer model for collaborative networks. Internet: http://www.olkc2013.com/sites/www.olk c2013.com/files/downloads/146.pdf, 2013, [February 20, 2015]

[12] D.G. Schwartz, Encyclopedia of Knowledge Management. Hershey, London, Melbourne, Singapore: Ed. Idea Group Reference, 2006, pp. 1-902.

[13] A. Capasso, G.B. Dagnino and A. Lanza, Strategic Capabilities and Knowledge Transfer Within and Between Organizations:New Perspectives from Acquisitions, Networks, Learning and Evolution, Ed. Edward Elgar Publishing, 2005, pp. 1-377.

[14] M. Brannback, "R\&D collaboration: role of $\mathrm{Ba}$ in knowledgecreating networks," Knowledge Management Research \& Practice, vol. 1, pp. 28-38, April 2003.

[15] A.A. Bolazeva. Inter-organizational knowledge transfer mechanisms in the focal company: a case study. Internet: http://dspace.ou.nl/bitstream/1820/3698/ 1/MWAABolazevamei2010.pdf, 2010 [February 20, 2015]

[16] W.F. Boh, "Mechanisms for Sharing Knowledge in Project-based Organizations," Information and Organization, vol. 17 , no. 1 , pp. 27-58, 2007.

[17] S.M. Wagner and C. Buko, "An Empirical Investigation of Knowledgesharing in Networks," The Journal of Supply Chain Management, vol. 41, no. 4, pp. 17-31, November 2005.

[18] B. Ghilic-Micu, M. Mircea, M. Stoica, "Performance indicators for virtual organizations in collaborative context", Proceedings of The 25th International Business Information Management Association Conference May 7-8, 2015, 
Amsterdam, Netherlands, Innovation

Economic Growth, Editor Khalid S. Vision 2020: From Regional

Development Sustainability to Global

Soliman, IBIMA 2015, pp. 712-720,

ISBN 978-0-9860419-4-5

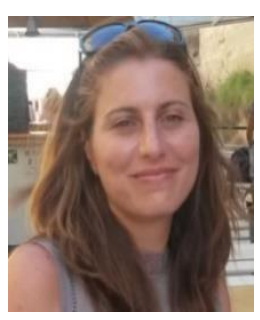

Marinela MIRCEA received her degree on Informatics in Economy from the Academy of Economic Studies, Bucharest in 2003 and his doctoral degree in economics in 2009. Since 2003 she is teaching in Academy of Economic Studies from Bucharest, at Informatics and Cybernetics Economy Department. Her work focuses on the programming, information system, business management and Business Intelligence. She published over 30 articles in journals and magazines in computer science, informatics and business management fields, over 30 papers presented at national and international conferences, symposiums and workshops and she was member over 15 research projects. She is the author and coauthor of 10 books. In February 2009, she finished the doctoral stage, and her $\mathrm{PhD}$ thesis has the title Business management in digital economy. 